\title{
Long-Time Behaviour of Solutions for Autonomous Evolution Hemivariational Inequality with Multidimensional "Reaction-Displacement" Law
}

\author{
Pavlo O. Kasyanov, ${ }^{1}$ Luisa Toscano, ${ }^{2}$ and Nina V. Zadoianchuk ${ }^{1}$ \\ ${ }^{1}$ Institute for Applied System Analysis NAS of Ukraine, Peremogy Avenue 37, Building 35, \\ Kyiv 03056, Ukraine \\ 2 Department of Mathematics and Applications "R. Caccioppoli", University of Naples "Federico II", \\ Via Claudio 21, 80125 Naples, Italy
}

Correspondence should be addressed to Pavlo O. Kasyanov, kasyanov@i.ua

Received 2 December 2011; Accepted 27 January 2012

Academic Editor: Muhammad Aslam Noor

Copyright (C) 2012 Pavlo O. Kasyanov et al. This is an open access article distributed under the Creative Commons Attribution License, which permits unrestricted use, distribution, and reproduction in any medium, provided the original work is properly cited.

We consider autonomous evolution inclusions and hemivariational inequalities with nonsmooth dependence between determinative parameters of a problem. The dynamics of all weak solutions defined on the positive semiaxis of time is studied. We prove the existence of trajectory and global attractors and investigate their structure. New properties of complete trajectories are justified. We study classes of mathematical models for geophysical processes and fields containing the multidimensional "reaction-displacement" law as one of possible application. The pointwise behavior of such problem solutions on attractor is described.

\section{Introduction}

Let a viscoelastic body occupies a bounded domain $\Omega \subset \mathbb{R}^{d}, d=2,3$ in applications, and it is acted upon by volume forces and surface tractions (this section is based on results of [1] and references therein). The boundary $\Gamma$ of $\Omega$ is supposed to be Lipschitz continuous, and it is partitioned into two disjoint measurable parts $\Gamma_{D}$ and $\Gamma_{N}$ such that means $\left(\Gamma_{D}\right)>0$. We consider the process of evolution of the mechanical state on the interval $(0,+\infty)$. The body is clamped on $\Gamma_{D}$ and thus the displacement vanishes there. The forces field of density $f_{0}$ act in $\Omega$, the surface tractions of density $g_{0}$ are applied on $\Gamma_{N}$. We denote by $u=\left(u_{1}, \ldots, u_{d}\right)$ the displacement vector, by $\sigma=\left(\sigma_{i j}\right)$ the stress tensor and by $\varepsilon(u)=\left(\varepsilon_{i j}(u)\right)$ the linearized (small) strain tensor $\left(\varepsilon_{i j}(u)=(1 / 2)\left(\partial_{j} u_{i}+\partial_{i} u_{j}\right)\right)$, where $i, j=1, \ldots, d$. 
The mechanical problem consists in finding the displacement field $u: \Omega \times(0,+\infty) \rightarrow$ $\mathbb{R}^{d}$ such that

$$
\begin{gathered}
u^{\prime \prime}(t)-\operatorname{div} \sigma(t)=f_{0} \quad \text { in } \Omega \times(0,+\infty), \\
\sigma(t)=\mathcal{C} \varepsilon\left(u^{\prime}(t)\right)+\varepsilon \varepsilon(u(t)) \quad \text { in } \Omega \times(0,+\infty), \\
u(t)=0 \quad \text { on } \Gamma_{D} \times(0,+\infty), \\
\sigma n(t)=g_{0} \quad \text { on } \Gamma_{N} \times(0,+\infty), \\
u(0)=u_{0}, \quad u^{\prime}(0)=u_{1} \quad \text { in } \Omega,
\end{gathered}
$$

where $\mathcal{C}$ and $\mathcal{E}$ are given linear constitutive functions and $n$ being the outward unit normal vector to $\Gamma$.

In the above model, the dynamic equation (1.1) is considered with the viscoelastic constitutive relationship of the Kelvin-Voigt type (1.2) while (1.3) and (1.4) represent the displacement and traction boundary conditions, respectively. The functions $u_{0}$ and $u_{1}$ are the initial displacement and the initial velocity, respectively. In order to formulate the skin effects, we suppose (following $[2,3]$ ) that the body forces of density $f_{0}$ consists of two parts: $f_{1}$ which is prescribed external loading and $f_{2}$ which is the reaction of constrains introducing the skin effects, that is, $f_{0}=f_{1}+f_{2}$. Here, $f_{2}$ is a possibly multivalued function of the displacement $u$. We consider the reaction-displacement law of the form:

$$
-f_{2}(x, t) \in \partial j(x, u(x, t)) \quad \text { in } \Omega \times(0,+\infty),
$$

where $j: \Omega \times \mathbb{R}^{d} \rightarrow \mathbb{R}$ is locally Lipschitz function in $u$, and $\partial j$ represents the Clarke subdifferential with respect to $u$. Let $y_{d}$ be the space of second-order symmetric tensors on $\mathbb{R}^{d}$.

We consider the following problem: examine the long-time (as $t \rightarrow+\infty$ ) behavior of all (weak, generalized) solutions for (1.1)-(1.5) and (1.6).

We remark that existence solutions theorems for evolution equations and inclusions where considered in [1-40] (see works and references therein).

In [1] for finite time interval, it was presented the hemivariational formulation of problems similar to (1.1)-(1.6) and an existence theorem for evolution inclusions with pseudomonotone operators. We give now variational formulation of the above problem. To this aim let $H=L_{2}\left(\Omega, \mathbb{R}^{d}\right), H_{1}=H^{1}\left(\Omega, \mathbb{R}^{d}\right), \mathscr{d}=L_{2}\left(\Omega, y_{d}\right)$, and $V$ be the closed subspace of $H_{1}$ defined by

$$
V=\left\{v \in H_{1}: v=0 \text { on } \Gamma_{D}\right\}
$$

On $V$ we consider the inner product and the corresponding norm given by

$$
(u, v)_{V}=\langle\varepsilon(u), \varepsilon(v)\rangle_{\mathscr{\ell}}, \quad\|v\|_{V}=\|\varepsilon(v)\|_{\mathscr{\ell}}, \quad \text { for } u, v \in V .
$$

From the Korn inequality $\|v\|_{H_{1}} \leq C_{1}\|\varepsilon(v)\|_{\mathscr{d}}$ for $v \in V$ with $C_{1}>0$, it follows that $\|\cdot\|_{H_{1}}$ and $\|\cdot\|_{V}$ are the equivalent norms on $V$. Identifying $H$ with its dual, we have 
an evolution triple $V \subset H \subset V^{*}$ (see, e.g., [8]) with dense and compact embeddings. We denote by $\langle\cdot, \cdot\rangle_{V}$ the duality of $V$ and its dual $V^{*}$, by $\|\cdot\|_{V^{*}}$ the norm in $V^{*}$. We have $\langle u, v\rangle_{V}=(u, v)_{H}$ for all $u \in H$ and $v \in V$.

We admit the following hypotheses.

$H(\mathcal{C})$ the linear symmetric viscosity operator $\mathcal{C}: \Omega \times y_{d} \rightarrow y_{d}$ satisfies the Carathéodory condition (i.e., $\mathcal{C}(\cdot, \varepsilon)$ is measurable on $\Omega$ for all $\varepsilon \in y_{d}$, and $\mathcal{C}(x, \cdot)$ is continuous on $y_{d}$ for a.e. $x \in \Omega$ ) and

$$
\mathcal{C}(x, \varepsilon): \varepsilon \geq C_{2}\|\varepsilon\|_{y_{d}}^{2} \quad \forall \varepsilon \in y_{d} \text {, a.e. } x \in \Omega \text { with } C_{2}>0 \text {. }
$$

$H(\varepsilon)$ the elasticity operator $\varepsilon: \Omega \times y_{d} \rightarrow y_{d}$ is of the form $\varepsilon(x, \varepsilon)=\mathbb{E}(x) \varepsilon$ (Hooke's law) with a symmetric elasticity tensor $\mathbb{E} \in L_{\infty}(\Omega)$, that is, $\mathbb{E}=\left(g_{i j k l}\right), i, j, k, l=1, \ldots, d$ with $g_{i j k l}=g_{j i k l}=g_{l k i j} \in L_{\infty}(\Omega)$. Moreover,

$$
\varepsilon(x, \varepsilon): \varepsilon \geq C_{3}\|\varepsilon\|_{y_{d}}^{2} \quad \forall \varepsilon \in y_{d} \text {, a.e. } x \in \Omega \text { with } C_{3}>0 \text {. }
$$

$H(j) j: \Omega \times \mathbb{R}^{d} \rightarrow \mathbb{R}$ is a function such that

(i) $j(\cdot, \xi)$ is measurable for all $\xi \in \mathbb{R}^{d}$ and $j(\cdot, 0) \in L_{1}(\Omega)$;

(ii) $j(x, \cdot)$ is locally Lipschitz and regular [5] for all $x \in \Omega$;

(iii) $\|\eta\| \leq C_{4}(1+\|\xi\|)$ for all $\eta \in \partial j(x, \xi), x \in \Omega$ with $C_{4}>0$;

(iv) $j^{0}(x, \xi ;-\xi) \leq C_{5}(1+\|\xi\|)$ for all $\xi \in \mathbb{R}^{d}, x \in \Omega$, with $C_{5} \geq 0$, where $j^{0}(x, \xi ; \eta)$ is the directional derivative of $j(x, \cdot)$ at the point $\xi \in \mathbb{R}^{d}$ in the direction $\eta \in \mathbb{R}^{d}$.

$H(f) f_{1} \in V^{*}, g_{0} \in L_{2}\left(\Gamma_{N} ; \mathbb{R}^{d}\right), u_{0} \in V$ and $u_{1} \in H$.

Next we need the spaces $\mathcal{U}=L_{2}(\tau, T ; V), \widehat{\mathscr{H}}=L_{2}(\tau, T ; H)$, and $\mathcal{W}=\left\{w \in \mathcal{V}: w^{\prime} \in \mathcal{U}^{*}\right\}$, where the time derivative involved in the definition of $\mathcal{W}$ is understood in the sense of vectorvalued distributions, $-\infty<\tau<T<+\infty$. Endowed with the norm $\|v\|_{\mathcal{W}}=\|v\|_{v}+\left\|v^{\prime}\right\|_{v^{*}}$, the space $\mathcal{W}$ becomes a separable reflexive Banach space. We also have $\mathcal{U} \subset \boldsymbol{V} \subset \widehat{\mathscr{d}} \subset v^{*}$. The duality for the pair $\left(\mho, \mho^{*}\right)$ is denoted by $\langle z, w\rangle_{\mho}=\int_{\tau}^{T}\langle z(s), w(s)\rangle_{V} d s$. It is well known (cf. [8]) that the embedding $\mathcal{W} \subset C([\tau, T] ; H)$ and $\left\{w \in \mathcal{V}: w^{\prime} \in \mathcal{W}\right\} \subset C([\tau, T] ; V)$ are continuous. Next we define $g \in V^{*}$ by

$$
\langle g, v\rangle_{V}=\left\langle f_{1}, v\right\rangle_{V}+\left\langle g_{0}, v\right\rangle_{L^{2}\left(\Gamma_{N} ; \mathbb{R}^{d}\right)} \quad \text { for } v \in V
$$

Taking into account the condition (1.6), we obtain the following variational formulation of our problem:

$$
\begin{gathered}
\left\langle u^{\prime \prime}(t), v\right\rangle_{V}+(\sigma(t), \varepsilon(v))_{\mathscr{l}}+\int_{\Omega} j^{0}(x, u(t) ; v) d x \geq\langle g, v\rangle_{V} \quad \forall v \in V, \text { a.e. } t \in(0,+\infty), \\
\sigma(t)=\mathcal{C}\left(\varepsilon\left(u^{\prime}(t)\right)\right)+\mathcal{E}(\varepsilon(u(t))) \text { for a.e. } t \in(0,+\infty), \\
u(0)=u_{0}, \quad u^{\prime}(0)=u_{1} .
\end{gathered}
$$


We define the operators $A: V \rightarrow V^{*}$ and $B: V \rightarrow V^{*}$ by

$$
\begin{array}{cc}
\langle A(u), v\rangle_{V}=(\mathcal{C}(x, \varepsilon(u)), \varepsilon(v))_{\mathscr{H}} & \text { for } u, v \in V, \\
\langle B u, v\rangle_{V}=(\mathcal{E}(x, \varepsilon(u)), \varepsilon(v))_{\mathscr{H}} & \text { for } u, v \in V .
\end{array}
$$

Obviously, the bilinear forms (1.13) are symmetric, continuous and coercive.

Let us introduce the functional $J: L_{2}\left(\Omega ; \mathbb{R}^{d}\right) \rightarrow \mathbb{R}$ defined by

$$
J(v)=\int_{\Omega} j(x, v(x)) d x \quad \text { for } v \in L_{2}\left(\Omega ; \mathbb{R}^{d}\right) .
$$

From [5, Chapter II] under Assumptions $H(j)$, the functional $J$ defined by (1.14) satisfies

$H(J) J: L_{2}\left(\Omega ; \mathbb{R}^{d}\right) \rightarrow \mathbb{R}$ is a functional such that:

(i) $J(\cdot)$ is well defined, locally Lipschitz (in fact, Lipschitz on bounded subsets of $\left.L_{2}\left(\Omega ; \mathbb{R}^{d}\right)\right)$ and regular;

(ii) $\zeta \in \partial J(v)$ implies $\|\zeta\|_{L_{2}\left(\Omega ; \mathbb{R}^{d}\right)} \leq C_{6}\left(1+\|v\|_{L_{2}\left(\Omega ; \mathbb{R}^{d}\right)}\right)$ for $v \in L_{2}\left(\Omega ; \mathbb{R}^{d}\right)$ with $C_{6}>0$;

(iii) $J^{0}(v ;-v) \leq C_{7}\left(1+\|v\|_{L_{2}\left(\Omega ; \mathbb{R}^{d}\right)}\right)$ for $v \in L_{2}\left(\Omega ; \mathbb{R}^{d}\right)$ with $C_{7} \geq 0$, where $J^{0}(u ; v)$ denotes the directional derivative of $J(\cdot)$ at a point $u \in L_{2}\left(\Omega ; \mathbb{R}^{d}\right)$ in the direction $v \in L_{2}\left(\Omega ; \mathbb{R}^{d}\right)$.

We can now formulate the second-order evolution inclusions associated with the variational form of our problem.

Find $u \in C([0,+\infty) ; V)$ with $u^{\prime} \in C([0,+\infty) ; H) \cap L_{2}^{\text {loc }}(0,+\infty ; V)$,

$$
u^{\prime \prime} \in L_{2}^{\text {loc }}\left(0,+\infty ; V^{*}\right)
$$

such that $u^{\prime \prime}(t)+A u^{\prime}(t)+B u(t)+\partial J(u(t)) \ni g$ a.e. $t \in(0,+\infty)$,

$$
u(0)=u_{0}, \quad u^{\prime}(0)=u_{1} .
$$

In this paper, we study a general autonomous evolution inclusion which includes (1.15).

\section{Setting of the Problem}

Let $V$ and $H$ be real separable Hilbert spaces such that $V \subset H$ with compact and dense embedding. Let $V^{*}$ be the dual space of $V$. We identify $H$ with $H^{*}$ (dual space of $H$ ). For the linear operators $A: V \rightarrow V^{*}, B: V \rightarrow V^{*}$, and locally Lipschitz functional $J: H \rightarrow \mathbb{R}$ we consider a problem of investigation of dynamics for all weak solutions defined for $t \geq 0$ of nonlinear second order autonomous differential-operator inclusion:

$$
u^{\prime \prime}(t)+A u^{\prime}(t)+B u(t)+\partial J(u(t)) \ni \overline{0}
$$


We need the following hypotheses

$H(A) A: V \rightarrow V^{*}$ is a linear symmetric such that there exists $c_{A}>0:\langle A \mathrm{v}, v\rangle_{V} \geq$ $\overline{c_{A}\|v\|_{V}^{2}}$ for all $v \in V$;

$H(B) B: V \rightarrow V^{*}$ is linear, symmetric, and there exists $c_{B}>0:\langle B v, v\rangle_{V} \geq$ $\overline{c_{B}\|v\|_{V}^{2}}$ for all $v \in V$ (we remark that operators $A$ and $B$ are continuous on $V$ $[8$, Chapter III]);

$\underline{H(J)} J: H \rightarrow \mathbf{R}$ is a function such that

(i) $J(\cdot)$ is locally Lipschitz and regular [5, Chapter II], that is,

(a) for any $x, v \in H$, the usual one-sided directional derivative $J^{\prime}(x ; v)=$ $\lim _{t \backslash 0}(J(x+t v)-J(x)) / t$ exists,

(b) for all $x, v \in H, J^{\prime}(x ; v)=J^{\circ}(x ; v)$, where $J^{\circ}(x ; v)=\varlimsup_{y \rightarrow x, t \searrow 0}(J(y+t v)-$ $J(y)) / t$;

(ii) there exists $c_{1}>0$ : $\sup _{d \in \partial J(v)}\|d\|_{H} \leq c_{1}\left(1+\|v\|_{H}\right)$ for all $v \in H$;

(iii) there exists $c_{2}>0$ :

$$
\inf _{d \in \partial J(v)}(d, v)_{H} \geq-\lambda\|v\|_{H}^{2}-c_{2}, \quad \forall v \in H
$$

where $\partial J(v)=\left\{p \in H \mid(p, w)_{H} \leq J^{\circ}(v ; w)\right.$ for all $\left.w \in H\right\}$ denotes the Clarke subdifferential of $J(\cdot)$ at a point $v \in H$ (see [5] for details), $\lambda \in\left(0, \lambda_{1}\right), \lambda_{1}>0$ : $\|v\|_{V}^{2} \geq \lambda_{1}\|v\|_{H}^{2}$ for all $v \in V$.

We note that in (1.15) we can consider $g=\overline{0}$. Indeed, let $g \in V^{*}$, then $u^{*}=B^{-1} g \in V \subset H$. If $u(\cdot)$ is a weak solution of $(1.15)$, then $\bar{u}(\cdot)=u(\cdot)-u^{*}$ is a weak solution of

$$
\bar{u}^{\prime \prime}(t)+A \bar{u}^{\prime}(t)+B \bar{u}(t)+\partial J_{1}(\bar{u}(t)) \ni \overline{0} \quad \text { a.e. } t>0,
$$

where $J_{1}(\cdot)=J\left(\cdot+u^{*}\right)$ satisfies $H(J)$ with respective parameters. Thus, to simplify our conclusions, without loss of generality, further we will consider problem (2.1).

The phase space for Problem (2.1) is the Hilbert space:

$$
E=\left\{(a, b)^{T} \mid a \in V, b \in H\right\}
$$

where $(a, b)^{T}=\left(\begin{array}{c}a \\ b\end{array}\right)$ with $\left\|(a, b)^{T}\right\|_{E}=\left(\|a\|_{V}^{2}+\|b\|_{H}^{2}\right)^{1 / 2}$. Let $-\infty<\tau<T<+\infty$.

Definition 2.1. The function $\left(u(\cdot), u^{\prime}(\cdot)\right)^{T} \in L_{\infty}(\tau, T ; E)$ with $u^{\prime}(\cdot) \in L_{2}(\tau, T ; V)$ is called a weak solution for (2.1) on $[\tau, T]$, if there exists $d(\cdot) \in L_{2}(\tau, T ; H), d(t) \in \partial J(u(t))$ for a.e. $t \in(\tau, T)$, such that for all $\psi \in V$, for all $\eta \in C_{0}^{\infty}(\tau, T)$ :

$$
-\int_{\tau}^{T}\left(u^{\prime}(t), \psi\right)_{H} \eta^{\prime}(t) d t+\int_{\tau}^{T}\left[\left\langle A u^{\prime}(t), \psi\right\rangle_{V}+\langle B u(t), \psi\rangle_{V}+(d(t), \psi)_{H}\right] \eta(t) d t=0 .
$$


Evidently, if $\left(u(\cdot), u^{\prime}(\cdot)\right)^{T}$ is a weak solution of $(2.1)$ on $[\tau, T]$, then $u^{\prime \prime}(\cdot) \in$ $L_{2}\left(\tau, T ; V^{*}\right),\left(u(\cdot), u^{\prime}(\cdot)\right)^{T} \in C([\tau, T] ; E)$ and $d(\cdot) \in L_{\infty}(\tau, T ; H)$.

We consider the class of functions $W_{\tau}^{T}=C([\tau, T] ; E)$. Further $c_{1}, c_{2}, \lambda, \lambda_{1}, c_{A}$, and $c_{B}$ we recall parameters of Problem (2.1). The main purpose of this work is to investigate the long-time behavior (as $t \rightarrow+\infty$ ) of all weak solutions for the problem $(2.1)$ on $[0,+\infty)$.

\section{Preliminaries}

To simplify our conclusions, since condition $\underline{H(B)}$, we suppose that

$$
(u, v)_{V}=\langle B u, v\rangle_{V}, \quad\|v\|_{V}^{2}=\langle B v, v\rangle_{V}, \quad c_{B}=1, \quad \forall u, v \in V
$$

Lebourg mean value theorem [5, Chapter 2] provides the existence of constants $c_{3}, c_{4}>0$ and $\mu \in\left(0, \lambda_{1}\right)$ :

$$
|J(u)| \leq c_{3}\left(1+\|u\|_{H}^{2}\right), \quad J(u) \geq-\frac{\mu}{2}\|u\|_{H}^{2}-c_{4}, \quad \forall u \in H .
$$

Lemma 3.1. Let $J: H \rightarrow \mathbb{R}$ be a locally Lipschitz and regular functional, $y \in C^{1}([\tau, T] ; H)$. Then for a.e. $t \in(\tau, T)$, there exists $(d / d t)(J \circ y)(t)=\left(p, y^{\prime}(t)\right)$ for all $p \in \partial J(y(t))$. Moreover, $(d / d t)(J \circ$ $y)(\cdot) \in L_{1}(\tau, T)$.

Proof. Since $y \in C^{1}([\tau, T] ; H)$, then $y$ is strictly differentiable at the point $t_{0}$ for any $t_{0} \in(\tau, T)$. Hence, taking into account the regularity of $J$ and [5, Theorem 2.3.10], we obtain that the functional $J \circ y$ is regular one at $t_{0} \in(\tau, T)$ and $\partial(J \circ y)\left(t_{0}\right)=\left\{\left(p, y^{\prime}\left(t_{0}\right)\right) \mid p \in \partial J\left(y\left(t_{0}\right)\right)\right\}$. On the other hand, since $y \in C^{1}([\tau, T] ; H), J: H \rightarrow \mathbb{R}$ is locally Lipschitz then $J \circ y:[\tau, T] \rightarrow \mathbb{R}$ is globally Lipschitz, and therefore it is absolutely continuous. Hence for a.e. $t \in(\tau, T)$ there exists $d(J \circ y)(t) / d t, d(J \circ y)(\cdot) / d t \in L_{1}(\tau, T)$, and $\int_{s}^{t}(d / d \xi)(J \circ y)(\xi) d \xi=(J \circ y)(t)-(J \circ y)(s)$ for all $\tau \leq s<t \leq T$. At that taking into account the regularity of $J \circ y$, note that $(J \circ y)^{\circ}\left(t_{0}, v\right)=$ $(J \circ y)^{\prime}\left(t_{0}, v\right)=d(J \circ y)\left(t_{0}\right) / d t \cdot v$ for a.e. $t_{0} \in(\tau, T)$, and all $v \in \mathbb{R}$. This implies that for a.e. $t_{0} \in(\tau, T) \partial(J \circ y)\left(t_{0}\right)=\left\{d(J \circ y)\left(t_{0}\right) / d t\right\}$.

At the inclusion (2.1) on $[\tau, T]$ we associate the conditions

$$
u(\tau)=a, \quad u^{\prime}(\tau)=b,
$$

where $a \in V$ and $b \in H$. From [37, Theorem 1] we get the following lemma.

Lemma 3.2. For any $\tau<T, a \in V, b \in H$ the Cauchy problem (2.1), (3.3) has a weak solution $\left(y, y^{\prime}\right)^{T} \in L_{\infty}(\tau, T ; E)$. Moreover, each weak solution $\left(y, y^{\prime}\right)^{T}$ of the Cauchy problem (2.1), (3.3) on the interval $[\tau, T]$ belongs to the space $C([\tau, T] ; E)$ and $y^{\prime} \in L_{2}(\tau, T ; V) ; y^{\prime \prime} \in L_{2}\left(\tau, T ; V^{*}\right)$. 


\section{Properties of Solutions}

Let us consider the next denotations: for all $\varphi_{\tau}=(a, b)^{T} \in E$ we set $\Phi_{\tau, T}\left(\varphi_{\tau}\right)=\left\{\left(u(\cdot), u^{\prime}(\cdot)\right)^{T}\right.$ | $\left(u, u^{\prime}\right)^{T}$ is a weak solution of $(2.1)$ on $[\tau, T], u(\tau)=a, u^{\prime}(\tau)=b$ \}. From Lemma 3.2, it follows that $\Phi_{\tau, T}\left(\varphi_{\tau}\right) \subset C([\tau, T] ; E)=W_{\tau}^{T}$.

Let us check that translation and concatenation of weak solutions are weak solutions too.

Lemma 4.1. If $\tau<T, \varphi_{\tau} \in E, \varphi(\cdot) \in \Phi_{\tau, T}\left(\varphi_{\tau}\right)$, then $\psi(\cdot)=\varphi(\cdot+s) \in \Phi_{\tau-s, T-s}\left(\varphi_{\tau}\right)$ for all $s$. If $\tau<t<T, \varphi_{\tau} \in E, \varphi(\cdot) \in \Phi_{\tau, t}\left(\varphi_{\tau}\right)$ and $\psi(\cdot) \in \Phi_{t, T}(\varphi(t))$, then

$$
\theta(s)= \begin{cases}\varphi(s), & s \in[\tau, t], \\ \psi(s), & s \in[t, T]\end{cases}
$$

belongs to $\bigoplus_{\tau, T}\left(\varphi_{\tau}\right)$.

Proof. The first part of the statement of this lemma follows from the autonomy of the inclusion (2.1). The proof of the second part follows from the definition of the solution of (2.1) and from that fact that $z \in W_{\tau, T}$ as soon as $v \in W_{\tau, t}, u \in W_{t, T}$ and $v(t)=u(t)$, where

$$
z(s)= \begin{cases}v(s), & s \in[\tau, t] \\ u(s), & s \in[t, T] .\end{cases}
$$

Let $\varphi=(a, b)^{T} \in E$ and

$$
\mho(\varphi)=\frac{1}{2}\|\varphi\|_{E}^{2}+J(a)
$$

Lemma 4.2. Let $\tau<T, \varphi_{\tau} \in E, \varphi(\cdot)=\left(y(\cdot), y^{\prime}(\cdot)\right)^{T} \in \Phi_{\tau, T}\left(\varphi_{\tau}\right)$. Then $\mho \circ \varphi:[\tau, T] \rightarrow \mathbb{R}$ is absolutely continuous and for a.e. $t \in(\tau, T) d \mho(\varphi(t)) / d t=-\left\langle A y^{\prime}(t), y^{\prime}(t)\right\rangle_{V} \leq-\gamma\left\|y^{\prime}(t)\right\|_{V}^{2}$, where $\gamma>0$ depends only on parameters of Problem (2.1) (we remark that $\sqrt{\langle A u, u\rangle_{V}}$ is equivalent norm on $V$, generated by inner product $\left.\langle A u, v\rangle_{V}\right)$.

Proof. Let $-\infty<\tau<T<+\infty, \varphi(\cdot)=\left(y(\cdot), y^{\prime}(\cdot)\right)^{T} \in W_{\tau}^{T}$ be an arbitrary weak solution of (2.1) on $(\tau, T)$. From [8, Chapter IV] we get that the function $t \rightarrow\left\|y^{\prime}(t)\right\|_{H}^{2}+\|y(t)\|_{V}^{2}$ is absolutely continuous and for a.e. $t \in(\tau, T)$ :

$$
\begin{aligned}
\frac{1}{2} \frac{d}{d t}\left[\left\|y^{\prime}(t)\right\|_{H}^{2}+\|y(t)\|_{V}^{2}\right] & =\left\langle y^{\prime \prime}(t)+B y(t), y^{\prime}(t)\right\rangle_{V}=-\left\langle A y^{\prime}(t), y^{\prime}(t)\right\rangle_{V}-\left(d(t), y^{\prime}(t)\right)_{H} \\
& \leq-\gamma\left\|y^{\prime}(t)\right\|_{V}^{2}-\left(d(t), y^{\prime}(t)\right)_{H^{\prime}}
\end{aligned}
$$

where $d(t) \in \partial J(y(t))$ for a.e. $t \in(\tau, T), d(\cdot) \in L_{2}(\tau, T ; H)$ and $\gamma>0$ depends only on parameters of Problem (2.1), in virtue of $u \rightarrow \sqrt{\langle A u, u\rangle_{V}}$ is equivalent norm on $V$. As $y(\cdot) \in$ $C^{1}([\tau, T] ; H)$ and $J: H \rightarrow \mathbb{R}$ is regular and locally Lipschitz, according to Lemma 3.1 we obtain that for a.e. $t \in(\tau, T)$ there exists $(d / d t)(J \circ y)(t)$. Moreover, $(d / d t)(J \circ y)(\cdot) \in L_{1}(\tau, T)$ 
and for a.e. $t \in(\tau, T)$ and all $p \in \partial J(y(t))$ we have $(d / d t)(J \circ y)(t)=\left(p, y^{\prime}(t)\right)_{H}$. In particular, for a.e. $t \in(\tau, T)(d / d t)(J \circ y)(t)=\left(d(t), y^{\prime}(t)\right)_{H}$. Taking into account (4.4), we finally obtain the necessary statement.

The lemma is proved.

Lemma 4.3. Let $T_{0}>0$. If $\left(u(\cdot), u^{\prime}(\cdot)\right)^{T}$ is a weak solution of $(2.1)$ on $\left[0, T_{0}\right]$, then there exists its extension on $[0,+\infty)\left(\bar{u}(\cdot), \bar{u}^{\prime}(\cdot)\right)^{T}$ which is weak solution of $(2.1)$ on $[0,+\infty)$, that is, $\left(\bar{u}(\cdot), \bar{u}^{\prime}(\cdot)\right)^{T} \in$ $C\left(\mathbb{R}_{+} ; E\right) \cap L_{\infty}\left(\mathbb{R}_{+} ; E\right)$ with $\bar{u}^{\prime}(\cdot) \in L_{2}(0, T ; V)$ for all $T>0$ and there exists $d(\cdot) \in L_{\infty}(0$, $+\infty ; H), d(t) \in \partial J(\bar{u}(t))$ for a.e. $t \in(0,+\infty)$, such that for all $\psi \in V$, for all $\eta \in C_{0}^{\infty}(0,+\infty)$ :

$$
-\int_{0}^{+\infty}\left(\bar{u}^{\prime}(t), \psi\right)_{H} \eta^{\prime}(t) d t+\int_{0}^{+\infty}\left[\left\langle A \bar{u}^{\prime}(t), \psi\right\rangle_{V}+\langle B \bar{u}(t), \psi\rangle_{V}+(d(t), \psi)_{H}\right] \eta(t) d t=0
$$

Proof. The statement of this lemma follows from Lemmas 3.2-4.2, Conditions (3.1), (3.2) and from the next estimates: for all $\tau<T$, for all $\varphi_{\tau} \in E$, for all $\varphi(\cdot)=\left(y(\cdot), y^{\prime}(\cdot)\right)^{T} \in \Phi_{\tau, T}\left(\varphi_{\tau}\right)$ for all $t \in[\tau, T] 2 c_{3}+\left(1+2 c_{3} / \lambda_{1}\right)\|y(\tau)\|_{V}^{2}+\left\|y^{\prime}(\tau)\right\|_{H}^{2} \geq 2 \mho(\varphi(\tau)) \geq 2 \mho(\varphi(t))=\|y(t)\|_{V}^{2}+\left\|y^{\prime}(t)\right\|_{H}^{2}+$ $2 J(y(t s)) \geq\left(1-\mu / \lambda_{1}\right)\|y(t)\|_{V}^{2}+\left\|y^{\prime}(t)\right\|_{H}^{2}-2 c_{4}$.

The lemma is proved.

For an arbitrary $\varphi_{0} \in E$, let $\Phi\left(\varphi_{0}\right)$ be the set of all weak solutions (defined on $[0,+\infty)$ ) of problem (2.1) with initial data $\varphi(0)=\varphi_{0}$. We remark that from the proof of Lemma 4.3 we obtain the next corollary.

Corollary 4.4. For any $\varphi_{0} \in E$ and $\varphi \in \Phi\left(\varphi_{0}\right)$ the next inequality is fulfilled:

$$
\|\varphi(t)\|_{E}^{2} \leq \frac{\lambda_{1}+2 c_{3}}{\lambda_{1}-\mu}\|\varphi(0)\|_{E}^{2}+\frac{2\left(c_{3}+c_{4}\right) \lambda_{1}}{\lambda_{1}-\mu} \quad \forall t>0
$$

From Corollary 4.4 and Conditions $H(A), H(B), H(J)$ in a standard way we obtain such proposition.

Theorem 4.5. Let $\tau<T,\left\{\varphi_{n}(\cdot)\right\}_{n \geq 1} \subset W_{\tau}^{T}$ be an arbitrary sequence of weak solutions of (2.1) on $[\tau, T]$ such that $\varphi_{n}(\tau) \rightarrow \varphi_{\tau}$ weakly in $E, n \rightarrow+\infty$. Then there exist $\varphi \in \Phi_{\tau, T}\left(\varphi_{\tau}\right)$ and $\left\{\varphi_{n_{k}}(\cdot)\right\}_{k \geq 1} \subset\left\{\varphi_{n}(\cdot)\right\}_{n \geq 1}$ such that $\varphi_{n_{k}}(\cdot) \rightarrow \varphi(\cdot)$ weakly in $E$ uniformly on $[\tau, T], k \rightarrow+\infty$, that is, $\varphi_{n_{k}}\left(t_{k}\right) \rightarrow \varphi\left(t_{0}\right)$ weakly in $E, k \rightarrow+\infty$ for any $\left\{t_{k}\right\}_{k \geq 1} \subset[\tau, T]$ with $t_{k} \rightarrow t_{0}, k \rightarrow+\infty$.

Theorem 4.6. Let $\tau<T,\left\{\varphi_{n}(\cdot)\right\}_{n \geq 1} \subset W_{\tau}^{T}$ be an arbitrary sequence of weak solutions of (2.1) on $[\tau, T]$ such that $\varphi_{n}(\tau) \rightarrow \varphi_{\tau}$ strongly in $E, n \rightarrow+\infty$. Then there exists $\varphi \in \Phi_{\tau, T}\left(\varphi_{\tau}\right)$ such that up to a subsequence $\varphi_{n}(\cdot) \rightarrow \varphi(\cdot)$ in $C([\tau, T] ; E), n \rightarrow+\infty$.

Proof. Let $\left\{\varphi_{n}(\cdot)=\left(u_{n}(\cdot), u_{n}^{\prime}(\cdot)\right)^{T}\right\}_{n \geq 1} \subset W_{\tau}^{T}$ be an arbitrary sequence of weak solutions of (2.1) on $[\tau, T]$ such that

$$
\varphi_{n}(\tau) \longrightarrow \varphi_{\tau} \text { strongly in } E, \quad n \rightarrow+\infty
$$


Let $\varphi=\left(u(\cdot), u^{\prime}(\cdot)\right)^{T} \in \Phi_{\tau, T}\left(\varphi_{\tau}\right)$ and $\left\{\varphi_{n_{k}}(\cdot)\right\}_{k \geq 1} \subseteq\left\{\varphi_{n}(\cdot)\right\}_{n \geq 1}$ as in Theorem 4.5. It is important to remark that in the proof of Theorem 4.5, by using the inequality (Lemma 4.2, Corollary 4.4, (3.2)):

$$
\gamma\left\|u_{n}^{\prime}(\cdot)\right\|_{L_{2}(\tau, T ; V)} \leq \mathcal{V}\left(\varphi_{n}(\tau)\right)-\mho\left(\varphi_{n}(T)\right) \leq \sup _{n \geq 1}\left[\mathcal{V}\left(\varphi_{n}(\tau)\right)+\frac{\mu}{2}\left\|u_{n}(T)\right\|_{H}^{2}\right]+c_{4}<+\infty
$$

we establish that

$$
u_{n_{k}}^{\prime}(\cdot) \longrightarrow u^{\prime}(\cdot) \text { weakly in } L_{2}(\tau, T ; V), \quad k \longrightarrow+\infty
$$

Let us prove that

$$
\varphi_{n_{k}} \longrightarrow \varphi \text { in } W_{\tau}^{T}, \quad k \longrightarrow+\infty
$$

By contradiction suppose the existence of $L>0$ and subsequence $\left\{\varphi_{k_{j}}\right\}_{j \geq 1} \subset\left\{\varphi_{n_{k}}\right\}_{k \geq 1}$ such that for all for all $j \geq 1 \max _{t \in[\tau, T]}\left\|\varphi_{k_{j}}(t)-\varphi(t)\right\|_{E}=\left\|\varphi_{k_{j}}\left(t_{j}\right)-\varphi\left(t_{j}\right)\right\|_{E} \geq L$. Without loss of generality we suppose that $t_{j} \rightarrow t_{0} \in[\tau, T], j \rightarrow+\infty$. Therefore, by virtue of the continuity of $\varphi:[\tau, T] \rightarrow E$, we have

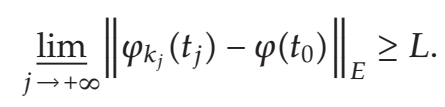

On the other hand, we prove that

$$
\varphi_{k_{j}}\left(t_{j}\right) \longrightarrow \varphi\left(t_{0}\right) \text { in } E, \quad j \longrightarrow+\infty
$$

Firstly, we remark that (Theorem 4.5)

$$
\varphi_{k_{j}}\left(t_{j}\right) \longrightarrow \varphi\left(t_{0}\right) \text { weakly in } E, \quad j \longrightarrow+\infty \text {. }
$$

Secondly, let us prove that

$$
\lim _{j \rightarrow+\infty}\left\|\varphi_{k_{j}}\left(t_{j}\right)\right\|_{E}=\left\|\varphi\left(t_{0}\right)\right\|_{E}
$$

Since $J$ is sequentially weakly continuous on $V, \mho$ is sequentially weakly lower semicontinuous on $E$. Hence, we obtain

$$
\mho\left(\varphi\left(t_{0}\right)\right) \leq \varliminf_{j \rightarrow+\infty} \mho\left(\varphi_{k_{j}}\left(t_{j}\right)\right), \quad \int_{\tau}^{t_{0}}\left\langle A u^{\prime}(s), u^{\prime}(s)\right\rangle_{V} d s \leq \varliminf_{j \rightarrow+\infty} \int_{\tau}^{t_{j}}\left\langle A u_{k_{j}}^{\prime}(s), u_{k_{j}}^{\prime}(s)\right\rangle_{V} d s,
$$


and hence

$$
\begin{aligned}
\mho\left(\varphi\left(t_{0}\right)\right)+\int_{\tau}^{t_{0}}\left\langle A u^{\prime}(s), u^{\prime}(s)\right\rangle_{V} d s & \leq \varliminf_{j \rightarrow+\infty} V\left(\varphi_{k_{j}}\left(t_{j}\right)\right)+\int_{\tau}^{t_{0}}\left\langle A u^{\prime}(s), u^{\prime}(s)\right\rangle_{V} d s \\
& \leq \varlimsup_{j \rightarrow+\infty} \mho\left(\varphi_{k_{j}}\left(t_{j}\right)\right)+\int_{\tau}^{t_{0}}\left\langle A u^{\prime}(s), u^{\prime}(s)\right\rangle_{V} d s \\
& \leq \varlimsup_{j \rightarrow+\infty} V\left(\varphi_{k_{j}}\left(t_{j}\right)\right)+\varliminf_{j \rightarrow+\infty} \int_{\tau}^{t_{j}}\left\langle A u_{k_{j}}^{\prime}(s), u_{k_{j}}^{\prime}(s)\right\rangle_{V} d s \\
& \leq \varlimsup_{j \rightarrow+\infty}\left(V\left(\varphi_{k_{j}}\left(t_{j}\right)\right)+\int_{\tau}^{t_{j}}\left\langle A u_{k_{j}}^{\prime}(s), u_{k_{j}}^{\prime}(s)\right\rangle_{V} d s\right) .
\end{aligned}
$$

We remark that the last inequality in (4.15) follows from weak convergence of $u_{n_{k}}^{\prime}(\cdot)$ to $u^{\prime}(\cdot)$ in $L_{2}(\tau, T ; V)$ and because of the functional $v \rightarrow \int_{\tau}^{T}\langle A v(s), v(s)\rangle_{V} d s$ is sequentially weakly lower semi-continuous on $L_{2}(\tau, T ; V)$.

Since the energy equation and (4.7) both sides of (4.16) are equal to $\mho(\varphi(\tau)$ ) (see Lemma 4.2), it follows that $\mho\left(\varphi_{k_{j}}\left(t_{j}\right)\right) \rightarrow \mho\left(\varphi\left(t_{0}\right)\right), j \rightarrow+\infty$ and then (4.14). Convergence (4.12) directly follows from (4.13) and (4.14). Finally, we remark that (4.12) contradicts (4.11). Therefore, (4.10) is true.

The theorem is proved.

We define the $m$-semiflow $\mathcal{G}_{1}$ as $\mathcal{G}_{1}\left(t, \xi_{0}\right)=\left\{\xi(t) \mid \xi(\cdot) \in \Phi\left(\xi_{0}\right)\right\}, t \geq 0$. Denote the set of all nonempty (nonempty bounded) subsets of $E$ by $P(E)(\beta(E)$ ). We remark that the multivalued map $\mathcal{G}_{1}: \mathbb{R}_{+} \times E \rightarrow P(E)$ is strict $m$-semiflow, that is, (see Lemma 4.1) $\mathcal{G}(0, \cdot)=\mathrm{Id}$ (the identity map), $\mathcal{G}(t+s, x)=\mathcal{G}(t, \mathcal{G}(s, x))$ for all $x \in E, t, s \in \mathbb{R}_{+}$. Further, $\varphi \in \mathcal{G}$ will mean that $\varphi \in \Phi\left(\xi_{0}\right)$ for some $\xi_{0} \in E$.

Definition 4.7 (see [41, page 35]). The $m$-semiflow $\mathcal{G}$ is called asymptotically compact, if for any sequence $\varphi_{j} \in \mathcal{G}$ with $\varphi_{j}(0)$ bounded, and for any sequence $t_{j} \rightarrow+\infty$, the sequence $\varphi_{j}\left(t_{j}\right)$ has a convergent subsequence.

Theorem 4.8. The m-semiflow $\mathcal{G}$ is asymptotically compact.

Proof. Let $\xi_{n} \in \mathcal{G}\left(t_{n}, v_{n}\right), v_{n} \in B \in \beta(E), n \geq 1, t_{n} \rightarrow+\infty, n \rightarrow+\infty$. Let us check the precompactness of $\left\{\xi_{n}\right\}_{n \geq 1}$ in $E$. In order to do that without loss of the generality it is sufficiently to extract a convergent in $E$ subsequence from $\left\{\xi_{n}\right\}_{n \geq 1}$. From Corollary 4.4 we obtain that there exist $\left\{\xi_{n_{k}}\right\}_{k \geq 1}$ and $\xi \in E$ such that $\xi_{n_{k}} \rightarrow \xi$ weakly in $E,\left\|\xi_{n_{k}}\right\|_{E} \rightarrow a \geq\|\xi\|_{E}$, $k \rightarrow+\infty$. We show that $a \leq\|\xi\|_{E}$. Let us fix an arbitrary $T_{0}>0$. Then for rather big $k \geq 1 \mathcal{G}\left(t_{n_{k}}, v_{n_{k}}\right)=\mathcal{G}\left(T_{0}, \mathcal{G}\left(t_{n_{k}}-T_{0}, v_{n_{k}}\right)\right)$. Hence $\xi_{n_{k}} \in \mathcal{G}\left(T_{0}, \beta_{n_{k}}\right)$, where $\beta_{n_{k}} \in \mathcal{G}_{\mathcal{G}}\left(t_{n_{k}}-T_{0}, v_{n_{k}}\right)$ and

$$
\delta:=\sup _{k \geq 1}\left\|\beta_{n_{k}}\right\|_{E}<+\infty
$$


(see Corollary 4.4). From Theorem 4.5 for some $\left\{\xi_{k_{j}}, \beta_{k_{j}}\right\}_{j \geq 1} \subset\left\{\xi_{n_{k}}, \beta_{n_{k}}\right\}_{k \geq 1}, \beta_{T_{0}} \in E$ we obtain

$$
\xi \in \mathcal{G}\left(T_{0}, \beta_{T_{0}}\right), \quad \beta_{k_{j}} \longrightarrow \beta_{T_{0}} \text { weakly in } E, \quad j \longrightarrow+\infty
$$

From the definition of $\mathcal{G}$ we set for all $j \geq 1 \xi_{k_{j}}=\left(y_{j}\left(T_{0}\right), y_{j}^{\prime}\left(T_{0}\right)\right)^{T}, \beta_{k_{j}}=\left(y_{j}(0), y_{j}^{\prime}(0)\right)^{T}, \xi=$ $\left(y_{0}\left(T_{0}\right), y_{0}^{\prime}\left(T_{0}\right)\right)^{T}, \beta_{T_{0}}=\left(y_{0}(0), y_{0}^{\prime}(0)\right)^{T}$, where $\varphi_{j}=\left(y_{j}, y_{j}^{\prime}\right)^{T} \in C\left(\left[0, T_{0}\right] ; E\right), y_{j}^{\prime} \in L_{2}\left(0, T_{0} ; V\right)$, $y_{j}^{\prime \prime} \in L_{2}\left(0, T_{0} ; V^{*}\right), d_{j} \in L_{\infty}\left(0, T_{0} ; H\right)$,

$$
y_{j}^{\prime \prime}(t)+A y_{j}^{\prime}(t)+B y_{j}(t)+d_{j}(t)=\overline{0}, \quad d_{j}(t) \in \partial J\left(y_{j}(t)\right) \quad \text { for a.e. } t \in\left(0, T_{0}\right), \forall j \geq 0
$$

Now we fix an arbitrary $\varepsilon>0$. Let for each $t \in\left[0, T_{0}\right], j \geq 0$ :

$$
I_{\varepsilon}\left(\varphi_{j}(t)\right):=\frac{1}{2}\left\|\varphi_{j}(t)\right\|_{E}^{2}+J\left(y_{j}(t)\right)+\varepsilon\left(y_{j}^{\prime}(t), y_{j}(t)\right)_{H} .
$$

Then, in virtue of $[8$, Chapter IV],

$$
\begin{aligned}
\frac{d I_{\varepsilon}\left(\varphi_{j}(t)\right)}{d t}= & -2 \varepsilon I_{\varepsilon}\left(\varphi_{j}(t)\right)+2 \varepsilon \mathscr{\ell}_{\varepsilon}\left(\varphi_{j}(t)\right)-\varepsilon\left\langle A y_{j}^{\prime}(t), y_{j}(t)\right\rangle_{V} \\
& -\left\langle A y_{j}^{\prime}(t), y_{j}^{\prime}(t)\right\rangle_{V^{\prime}} \text { for a.e. } t \in\left(0, T_{0}\right),
\end{aligned}
$$

where

$$
\begin{aligned}
\mathscr{L}_{\varepsilon}\left(\varphi_{j}(t)\right)= & J\left(y_{j}(t)\right)-\frac{1}{2}\left(d_{j}(t), y_{j}(t)\right)_{H}+\left\|y_{j}^{\prime}(t)\right\|_{H}^{2} \\
& +\varepsilon\left(y_{j}^{\prime}(t), y_{j}(t)\right)_{H^{\prime}} \quad \text { for a.e. } t \in\left(0, T_{0}\right) .
\end{aligned}
$$

Thus, for any $j \geq 0$ and $t \in\left[0, T_{0}\right]$

$$
\begin{aligned}
I_{\varepsilon}\left(\varphi_{j}\left(T_{0}\right)\right)= & I_{\varepsilon}\left(\varphi_{j}(0)\right) e^{-2 \varepsilon T_{0}}+2 \varepsilon \int_{0}^{T_{0}} \mathscr{\ell}_{\varepsilon}\left(\varphi_{j}(t)\right) e^{-2 \varepsilon\left(T_{0}-t\right)} d t \\
& -\varepsilon \int_{0}^{T_{0}}\left\langle A y_{j}(t), y_{j}^{\prime}(t)\right\rangle_{V} e^{-2 \varepsilon\left(T_{0}-t\right)} d t-\int_{0}^{T_{0}}\left\langle A y_{j}^{\prime}(t), y_{j}^{\prime}(t)\right\rangle_{V} e^{-2 \varepsilon\left(T_{0}-t\right)} d t .
\end{aligned}
$$

From (4.6), (4.18) and Lemma 4.2 we have

$$
\left\|y_{j}^{\prime}(t)\right\|_{H}^{2}+\left\|y_{j}(t)\right\|_{V}^{2}+\gamma \int_{0}^{t}\left\|y_{j}^{\prime}(t)\right\|_{V}^{2} d t \leq \bar{R}, \quad \forall j \geq 0, \forall t \in\left[0, T_{0}\right]
$$


where $\bar{R}>0$ is a constant. Moreover,

$$
\begin{aligned}
& y_{j} \longrightarrow y_{0} \text { weakly in } L_{2}\left(0, T_{0} ; V\right), y_{j}^{\prime} \longrightarrow y_{0}^{\prime} \text { weakly in } L_{2}\left(0, T_{0} ; V\right), \\
& y_{j} \longrightarrow y_{0} \text { strongly in } L_{2}\left(0, T_{0} ; H\right), y_{j}^{\prime} \longrightarrow y_{0}^{\prime} \text { strongly in } L_{2}\left(0, T_{0} ; H\right), \\
& y_{j}^{\prime \prime} \longrightarrow y_{0}^{\prime \prime} \text { weakly in } L_{2}\left(0, T_{0} ; V^{*}\right), d_{j} \longrightarrow d_{0} \text { weakly in } L_{2}\left(0, T_{0} ; H\right), \\
& \forall t \in\left[0, T_{0}\right] \quad y_{j}(t) \longrightarrow y_{0}(t) \text { in } H, \quad y_{j}^{\prime}(t) \longrightarrow y_{0}^{\prime}(t) \text { weakly in } H, \quad j \longrightarrow+\infty .
\end{aligned}
$$

Therefore,

$$
\begin{gathered}
\int_{0}^{T_{0}} \mathscr{L}_{\varepsilon}\left(\varphi_{j}(t)\right) e^{-2 \varepsilon\left(T_{0}-t\right)} d t \longrightarrow \int_{0}^{T_{0}} \mathscr{L}_{\varepsilon}\left(\varphi_{0}(t)\right) e^{-2 \varepsilon\left(T_{0}-t\right)} d t, \quad j \longrightarrow+\infty, \\
\lim _{j \rightarrow+\infty} \int_{0}^{T_{0}}\left\langle A y_{j}^{\prime}(t), y_{j}^{\prime}(t)\right\rangle_{V} e^{-2 \varepsilon\left(T_{0}-t\right)} d t \geq \int_{0}^{T_{0}}\left\langle A y_{0}^{\prime}(t), y_{0}^{\prime}(t)\right\rangle_{V} e^{-2 \varepsilon\left(T_{0}-t\right)} d t .
\end{gathered}
$$

The last inequality holds because of the functional $v(\cdot) \rightarrow \int_{0}^{T_{0}}\langle A v(t), v(t)\rangle_{V} e^{-2 \varepsilon\left(T_{0}-t\right)} d t$ is sequentially weakly lower semicontinuous on $L_{2}\left(\tau, T_{0} ; V\right)$. Furthermore,

$$
\begin{aligned}
\varepsilon \int_{0}^{T_{0}}\left\langle A y_{j}(t), y_{j}^{\prime}(t)\right\rangle_{V} e^{-2 \varepsilon\left(T_{0}-t\right)} d t= & \frac{\varepsilon}{2}\left\langle A y_{j}\left(T_{0}\right), y_{j}\left(T_{0}\right)\right\rangle_{V}-\frac{\varepsilon}{2}\left\langle A y_{j}(0), y_{j}(0)\right\rangle_{V} e^{-2 \varepsilon T_{0}} \\
& -\varepsilon^{2} \int_{0}^{T_{0}}\left\langle A y_{j}(t), y_{j}(t)\right\rangle_{V} e^{-2 \varepsilon\left(T_{0}-t\right)} d t \quad \forall j \geq 0
\end{aligned}
$$

from which, by Corollary 4.4, we have

$$
\left|\varepsilon \int_{0}^{T_{0}}\left\langle A y_{j}(t), y_{j}^{\prime}(t)\right\rangle_{V} e^{-2 \varepsilon\left(T_{0}-t\right)} d t\right| \leq 2 \varepsilon \gamma\left[\frac{\lambda_{1}+2 c_{3}}{\lambda_{1}-\mu} \delta^{2}+\frac{2\left(c_{3}+c_{4}\right) \lambda_{1}}{\lambda_{1}-\mu}\right], \quad \forall j \geq 0
$$

Thus,

$$
\begin{aligned}
\varlimsup_{j \rightarrow+\infty} I_{\varepsilon}\left(\varphi_{j}\left(T_{0}\right)\right) \leq & I_{\varepsilon}\left(\varphi_{0}(0)\right) e^{-2 \varepsilon T_{0}}+\left[\varlimsup_{j \rightarrow+\infty} I_{\varepsilon}\left(\varphi_{j}(0)\right)-I_{\varepsilon}\left(\varphi_{0}(0)\right)\right] e^{-2 \varepsilon T_{0}} \\
& +2 \varepsilon \int_{0}^{T_{0}} \mathscr{L}_{\varepsilon}\left(\varphi_{0}(t)\right) e^{-2 \varepsilon\left(T_{0}-t\right)} d t-\varepsilon \int_{0}^{T_{0}}\left\langle A y_{0}(t), y_{0}^{\prime}(t)\right\rangle_{V} e^{-2 \varepsilon\left(T_{0}-t\right)} d t \\
& -\int_{0}^{T_{0}}\left\langle A y_{0}^{\prime}(t), y_{0}^{\prime}(t)\right\rangle_{V} e^{-2 \varepsilon\left(T_{0}-t\right)} d t+4 \varepsilon \gamma\left[\frac{\lambda_{1}+2 c_{3}}{\lambda_{1}-\mu} \delta^{2}+\frac{2\left(c_{3}+c_{4}\right) \lambda_{1}}{\lambda_{1}-\mu}\right] \\
\leq & I_{\varepsilon}\left(\varphi_{0}\left(T_{0}\right)\right)+\delta^{2} e^{-2 \varepsilon T_{0}}+4 \varepsilon \gamma\left[\frac{\lambda_{1}+2 c_{3}}{\lambda_{1}-\mu} \delta^{2}+\frac{2\left(c_{3}+c_{4}\right) \lambda_{1}}{\lambda_{1}-\mu}\right],
\end{aligned}
$$


and, due to (4.25), for any $T_{0}>0$ and $\varepsilon>0$

$$
\frac{1}{2}\|\xi\|_{E}^{2} \leq \frac{1}{2} a^{2}=\frac{1}{2} \lim _{j \rightarrow+\infty}\left\|\xi k_{j}\right\|_{E}^{2} \leq \frac{1}{2}\|\xi\|_{E}^{2}+\delta^{2} e^{-2 \varepsilon T_{0}}+4 \varepsilon \gamma\left[\frac{\lambda_{1}+2 c_{3}}{\lambda_{1}-\mu} \delta^{2}+\frac{2\left(c_{3}+c_{4}\right) \lambda_{1}}{\lambda_{1}-\mu}\right] .
$$

Hence, for all $\varepsilon>0$ we have

$$
\frac{1}{2}\|\xi\|_{E}^{2} \leq \frac{1}{2} a^{2} \leq \frac{1}{2}\|\xi\|_{E}^{2}+4 \varepsilon \gamma\left[\frac{\lambda_{1}+2 c_{3}}{\lambda_{1}-\mu} \delta^{2}+\frac{2\left(c_{3}+c_{4}\right) \lambda_{1}}{\lambda_{1}-\mu}\right] .
$$

Thus, $a=\|\xi\|_{E}$.

The theorem is proved.

Let us consider the family $\mathcal{K}_{+}=\cup_{y_{0} \in E} \boldsymbol{\Phi}\left(y_{0}\right)$ of all weak solutions of the inclusion (2.1), defined on $[0,+\infty)$. Note that $\mathcal{K}_{+}$is translation invariant one, that is, for all $u(\cdot) \in \mathcal{K}_{+}$and all $h \geq 0$ we have $u_{h}(\cdot) \in \mathcal{K}_{+}$, where $u_{h}(s)=u(h+s), s \geq 0$. On $\mathcal{K}_{+}$, we set the translation semigroup $\{T(h)\}_{h \geq 0}, T(h) u(\cdot)=u_{h}(\cdot), h \geq 0, u \in \mathcal{K}_{+}$. In view of the translation invariance of $\mathcal{K}_{+}$, we conclude that $T(h) \mathcal{K}_{+} \subset \mathcal{K}_{+}$as $h \geq 0$.

On $\mathcal{K}_{+}$, we consider the topology induced from the Fréchet space $C^{\text {loc }}\left(\mathbb{R}_{+} ; E\right)$. Note that

$$
f_{n}(\cdot) \longrightarrow f(\cdot) \text { in } C^{\text {loc }}\left(\mathbb{R}_{+} ; E\right) \Longleftrightarrow \forall M>0 \quad \Pi_{M} f_{n}(\cdot) \longrightarrow \Pi_{M} f(\cdot) \text { in } C([0, M] ; E),
$$

where $\Pi_{M}$ is the restriction operator to the interval $[0, M]$ [42, page 179]. We denote the restriction operator to $[0,+\infty)$ by $\Pi_{+}$.

Let us consider the autonomous inclusion (2.1) on the entire time axis. Similarly to the space $C^{\text {loc }}\left(\mathbb{R}_{+} ; E\right)$, the space $C^{\text {loc }}(\mathbb{R} ; E)$ is endowed with the topology of local uniform convergence on each interval $[-M, M] \subset \mathbb{R}$ (cf. [42, page 180]). A function $u \in C^{\text {loc }}(\mathbb{R} ; E) \cap$ $L_{\infty}(\mathbb{R} ; E)$ is said to be a complete trajectory of the inclusion $(2.1)$, if for all $h \in \mathbb{R} \Pi_{+} u_{h}(\cdot) \in \mathcal{K}_{+}$ [42, page 180]. Let $\mathcal{K}$ be a family of all complete trajectories of the inclusion (2.1). Note that for all $h \in \mathbb{R}$, for all $u(\cdot) \in \mathcal{K} u_{h}(\cdot) \in \mathcal{K}$. We say that the complete trajectory $\varphi \in \mathcal{K}$ is stationary if $\varphi(t)=z$ for all $t \in \mathbb{R}$ for some $z=(u, \overline{0})^{T} \in E$ (rest point). Following [43, page 486], we denote the set of rest points of $\mathcal{G}$ by $Z(\mathcal{G})$. We remark that $Z(\mathcal{G})=\left\{(u, \overline{0})^{T} \mid u \in V, B(u)+\partial J(u) \ni \overline{0}\right\}$.

From Conditions $H(B)$ and $H(J)$ and [39, Chapter 2], the following follows.

Lemma 4.9. The set $Z(\mathcal{G})$ is nonempty and bounded in $E$.

From Lemma 4.2, the existence of Lyapunov function (see [43, page 486]) for $\mathcal{G}$ follows.

Lemma 4.10. The functional $\mho: E \rightarrow \mathbb{R}$, defined by (4.3), is a Lyapunov function for $\mathcal{G}$. 


\section{Existence of the Global Attractor}

First we consider constructions presented in $[43,44]$. We recall that the set $\mathcal{A} \subset E$ is said to be a global attractor for $\mathcal{G}$, if

(1) $\mathcal{A}$ is negatively semi-invariant (i.e., $\mathscr{A} \subset \mathcal{G}(t, \mathcal{A})$ for all $t \geq 0$ );

(2) A is attracting set, that is,

$$
\operatorname{dist}(\mathcal{G}(t, B), \mathcal{A}) \longrightarrow 0, \quad t \longrightarrow+\infty \forall B \in \beta(E),
$$

where $\operatorname{dist}(C, D)=\sup _{c \in C} \inf _{d \in D}\|c-d\|_{E}$ is the Hausdorff semidistance;

(3) for any closed set $Y \subset E$, satisfying (5.1), we have $\mathcal{A} \subset Y$ (minimality).

The global attractor is said to be invariant, if $\mathbb{A}=\mathcal{G}(t, \mathcal{A})$ for all $t \geq 0$.

Note that from the definition of the global attractor it follows that it is unique.

We prove the existence of the invariant compact global attractor.

Theorem 5.1. The $m$-semiflow $\mathcal{G}$ has the invariant compact in the phase space E global attractor $A$. For each $\psi \in \mathcal{K}$ the limit sets:

$$
\begin{aligned}
& \alpha(\psi)=\left\{z \in E \mid \psi\left(t_{j}\right) \longrightarrow z \text { for some sequence } t_{j} \longrightarrow-\infty\right\}, \\
& \omega(\psi)=\left\{z \in E \mid \psi\left(t_{j}\right) \longrightarrow z \text { for some sequence } t_{j} \longrightarrow+\infty\right\},
\end{aligned}
$$

are connected subsets of $Z(\mathcal{G})$ on which $\mho$ is constant. If $Z(\mathcal{G})$ is totally disconnected (in particular, if $Z(\mathcal{G})$ is countable) the limits:

$$
z_{-}=\lim _{t \rightarrow-\infty} \psi(t), \quad z_{+}=\lim _{t \rightarrow+\infty} \psi(t)
$$

exist; $z_{-}$and $z_{+}$are rest points; furthermore, $\varphi(t)$ tends to a rest point as $t \rightarrow+\infty$ for every $\varphi \in \mathcal{K}_{+}$.

Proof. The existence of the global attractor with required properties directly follows from Lemmas 3.2, 4.1, 4.9, and 4.10, Theorems 4.5-4.8 and [41, Theorem 2.7].

\section{Properties of Complete Trajectories}

We remark in advance (Section 4):

$$
\forall h \in \mathbb{R}, \forall u(\cdot) \in \mathcal{K} \quad u_{h}(\cdot) \in \mathcal{K} .
$$

Lemma 6.1. The set $\mathcal{K}$ is nonempty, and

$$
\forall \xi(\cdot) \in \mathcal{K}, \forall t \in \mathbb{R} \quad \xi(t) \in \mathcal{A},
$$

where $A$ is the global attractor from Theorem 5.1. 
Proof. Let us show that $\mathcal{K} \neq \emptyset$. Note that in virtue of Lemma 4.9 , the set $Z(\mathcal{G})$ is nonempty and bounded in $E$. Let $(v, \overline{0})^{T} \in Z(\mathcal{G})$. We set $u(t)=v$ for all $t \in \mathbb{R}$. Then $\left(u, u^{\prime}\right)^{T} \in \mathcal{K} \neq \emptyset$.

Let us prove (6.2). For any $y \in \mathcal{K}$ there exists $d>0:\|y(t)\|_{E} \leq d$ for all $t \in \mathbb{R}$. We set $B=\cup_{t \in \mathbb{R}}\{y(t)\} \in \beta(E)$. Note that for all $\tau \in \mathbb{R}$, for all $t \in \mathbb{R}_{+} y(\tau)=y_{\tau-t}(t) \in \mathcal{G}\left(t, y_{\tau-t}(0)\right) \subset$ $\mathcal{G}(t, B)$. From Theorem 5.1 and from (5.1), it follows that for all $\varepsilon>0$ there exists $T>0$ : for all $\tau \in \mathbb{R} \operatorname{dist}(y(\tau), \mathcal{A}) \leq \operatorname{dist}(\mathcal{G}(T, B), \mathcal{A})<\varepsilon$. Hence taking into account the compactness of $\mathcal{A}$ in $E$, it follows that $y(\tau) \in \mathcal{A}$ for any $\tau \in \mathbb{R}$.

Lemma 6.2. The set $\mathcal{K}$ is compact in $C^{\text {loc }}(\mathbb{R} ; E)$ and bounded in $L_{\infty}(\mathbb{R} ; E)$.

Proof. The boundedness of $\mathcal{K}$ in $L_{\infty}\left(\mathbb{R}_{+} ; E\right)$ follows from (6.2) and from the boundedness of $A$ in $E$.

Let us check the compactness of $\mathcal{K}$ in $C^{\text {loc }}(\mathbb{R} ; E)$. In order to do that it is sufficient to check the precompactness and completeness.

Step 1. Let us check the precompactness of $\mathcal{K}$ in $C^{\text {loc }}(\mathbb{R} ; E)$. If it is not true then in view of (6.1), there exists $M>0: \Pi_{M} \mathcal{K}$ is not precompact set in $C([0, M] ; E)$. Hence there exists a sequence $\left\{v_{n}\right\}_{n \geq 1} \subset \Pi_{M} \mathcal{K}$, that has not a convergent subsequence in $C([0, M] ; E)$. On the other hand $v_{n}=\Pi_{M} u_{n}$, where $u_{n} \in \mathcal{K}, v_{n}(0)=u_{n}(0) \in \mathcal{A}, n \geq 1$. Since $\mathcal{A}$ is compact set in $E$ (see Theorem 5.1), then in view of Theorem 4.6, there exists $\left\{v_{n_{k}}\right\}_{k \geq 1} \subset\left\{v_{n}\right\}_{n \geq 1}$, there exists $\eta \in E$, there exists $v(\cdot) \in \Phi_{0, M}(\eta): v_{n_{k}}(0) \rightarrow \eta$ in $E, v_{n_{k}} \rightarrow v$ in $C([0, T] ; E), k \rightarrow+\infty$. We obtained a contradiction.

Step 2. Let us check the completeness of $\mathcal{K}$ in $C^{\text {loc }}(\mathbb{R} ; E)$. Let $\left\{v_{n}\right\}_{n \geq 1} \subset \mathcal{K}, v \in C^{\text {loc }}(\mathbb{R} ; E)$ : $v_{n} \rightarrow v$ in $C^{\text {loc }}(\mathbb{R} ; E), n \rightarrow+\infty$. From the boundedness of $\mathcal{K}$ in $L_{\infty}(\mathbb{R} ; E)$, it follows that $v \in L_{\infty}(\mathbb{R} ; E)$. From Theorem 4.6 we have that for all $M>0$ the restriction $v(\cdot)$ to the interval $[-M, M]$ belongs to $\Phi_{-M, M}(v(-M))$. Therefore, $v(\cdot)$ is a complete trajectory of the inclusion (2.1). Thus, $v \in \mathcal{K}$.

Lemma 6.3. Let $A$ be the global attractor from Theorem 5.1. Then

$$
\forall y_{0} \in \mathcal{A} \exists y(\cdot) \in \mathcal{K}: \quad y(0)=y_{0}
$$

Proof. Let $y_{0} \in \mathcal{A}, u(\cdot) \in \mathscr{\Phi}\left(y_{0}\right)$. From Theorem $5.1 \mathcal{G}(t, \mathcal{A})=\mathscr{A}$ for all $t \in \mathbb{R}_{+}$. Therefore,

$$
\begin{gathered}
u(t) \in \mathcal{A} \quad \forall t \in \mathbb{R}_{+}, \\
\forall \eta \in \mathcal{A} \exists \xi \in \mathcal{A}, \exists \varphi_{\eta}(\cdot) \in \mathscr{\Phi}_{0,1}(\xi): \quad \varphi_{\eta}(1)=\eta .
\end{gathered}
$$

For any $t \in \mathbb{R}$, we set

$$
y(t)= \begin{cases}u(t), & t \in \mathbb{R}_{+} \\ \varphi_{y(-k+1)}(t+k), & t \in[-k,-k+1), k \in \mathbb{N} .\end{cases}
$$

Note that $y \in C^{\text {loc }}(\mathbb{R} ; E), y(t) \in \mathcal{A}$ for all $t \in \mathbb{R}$ (hence $y \in L_{\infty}(\mathbb{R} ; E)$ ) and in view of Lemma 4.1, $y \in \mathcal{K}$. Moreover $y(0)=y_{0}$. 


\section{Existence of the Trajectory Attractor}

We shall construct the attractor of the translation semigroup $\{T(h)\}_{h \geq 0}$, acting on $\mathcal{K}_{+}$. We recall that the set $D \subset C^{\text {loc }}\left(\mathbb{R}_{+} ; E\right) \cap L_{\infty}\left(\mathbb{R}_{+} ; E\right)$ is said to be an attracting one for the trajectory space $\mathcal{K}_{+}$of the inclusion (2.1) in the topology of $C^{\text {loc }}\left(\mathbb{R}_{+} ; E\right)$, if for any bounded (in $\left.L_{\infty}\left(\mathbb{R}_{+} ; E\right)\right)$ set $B \subset \mathcal{K}_{+}$and an arbitrary number $M \geq 0$ the next relation:

$$
\operatorname{dist}_{C([0, M] ; E)}\left(\Pi_{M} T(t) B, \Pi_{M} D\right) \longrightarrow 0, \quad t \longrightarrow+\infty
$$

holds. A set $U \subset \mathcal{K}_{+}$is said to be trajectory attractor in the trajectory space $\mathcal{K}_{+}$with respect to the topology of $C^{\text {loc }}\left(\mathbb{R}_{+} ; E\right.$ ) (cf. [42], Definition 1.2, page 179), if

(i) $\mathcal{U}$ is a compact set in $C^{\text {loc }}\left(\mathbb{R}_{+} ; E\right)$ and bounded in $L_{\infty}\left(\mathbb{R}_{+} ; E\right)$;

(ii) $\mathcal{U}$ is strictly invariant with respect to $\{T(h)\}_{h \geq 0}$, that is, $T(h) \mathcal{U}=\mathfrak{U}$ for all $h \geq 0$;

(iii) $\mathcal{U}$ is an attracting set in the trajectory space $\mathcal{K}_{+}$in the topology $C^{\text {loc }}\left(\mathbb{R}_{+} ; E\right)$.

Note that from the definition of the trajectory attractor it follows that it is unique.

The existence of the trajectory attractor and its structure properties follow from such theorem.

Theorem 7.1. Let $A$ be the global attractor from Theorem 5.1. Then there exists the trajectory attractor $D \subset \mathcal{K}_{+}$in the space $\mathcal{K}_{+}$, and we have

$$
p=\Pi_{+} \mathcal{K}=\left\{y \in \mathcal{K}_{+} \mid y(t) \in \mathcal{A} \forall t \in \mathbb{R}_{+}\right\} .
$$

Proof. The proof intersects with proofs of corresponding statements from [14, 45] (see papers and references therein), and it is based on previous sections results.

From Lemmas 6.1 and 6.2 and the continuity of the operator $\Pi_{+}: C^{\text {loc }}(\mathbb{R} ; E) \rightarrow$ $C^{\text {loc }}\left(\mathbb{R}_{+} ; E\right)$ it follows that the set $\Pi_{+} \mathcal{K}$ is nonempty, compact in $C^{\text {loc }}\left(\mathbb{R}_{+} ; E\right)$ and bounded in $L_{\infty}\left(\mathbb{R}_{+} ; E\right)$. Moreover, the second equality in (7.2) holds (Lemma 6.1 and the proof of Lemma 6.3). The strict invariance of $\Pi_{+} \mathcal{K}$ follows from the autonomy of the inclusion (2.1).

Let us prove that $\Pi_{+} \mathcal{K}$ is the attracting set for the trajectory space $\mathcal{K}_{+}$in the topology of $C^{\text {loc }}\left(\mathbb{R}_{+} ; E\right)$. Let $B \subset \mathcal{K}_{+}$be a bounded set in $L_{\infty}\left(\mathbb{R}_{+} ; E\right), M \geq 0$. Let us suppose $M>0$. Let us check (7.1). If it is not true, then there exist $\varepsilon>0$, the sequences $t_{n} \rightarrow+\infty, v_{n}(\cdot) \in B$ such that

$$
\forall n \geq 1 \quad \operatorname{dist}_{C([0, T] ; E)}\left(\Pi_{M} v_{n}\left(t_{n}+\cdot\right), \Pi_{M} \mathcal{K}\right) \geq \varepsilon
$$

On the other hand, from the boundedness of $B$ in $L_{\infty}\left(\mathbb{R}_{+} ; E\right)$ it follows that there exists $R>0$ : for all $v(\cdot) \in B$, for all $t \in \mathbb{R}_{+}\|v(t)\|_{E} \leq R$. Hence, taking into account (5.1) and the asymptotic compactness of $m$-semiflow $\mathcal{G}$ (Theorem 4.8), we obtain that there exists $\left\{v_{n_{k}}\left(t_{n_{k}}\right)\right\}_{k>1} \subset$ $\left\{v_{n}\left(t_{n}\right)\right\}_{n>1}$, there exists there exists $z \in \mathcal{A}: v_{n_{k}}\left(t_{n_{k}}\right) \rightarrow z$ in $E, k \rightarrow+\infty$. Further, for all $k \geq 1$ we set $\varphi_{k}(t)=v_{n_{k}}\left(t_{n_{k}}+t\right), t \in[0, M]$. Note that for all $k \geq 1 \varphi_{k}(\cdot) \in \Phi_{0, M}\left(v_{n_{k}}\left(t_{n_{k}}\right)\right)$. Then from Theorem 4.6 there exists a subsequence $\left\{\varphi_{k_{j}}\right\}_{j \geq 1} \subset\left\{\varphi_{k}\right\}_{k \geq 1}$ and an element $\varphi(\cdot) \in \Phi_{0, M}(z)$ :

$$
\varphi_{k_{j}} \longrightarrow \varphi \text { in } C([0, M] ; E), \quad j \longrightarrow+\infty
$$


Moreover, taking into account the invariance of $\mathcal{A}$ (see Theorem 5.1) for all $t \in[0, M] \varphi(t) \in$ A. From Lemma 6.3, there exist $y(\cdot), v(\cdot) \in \mathcal{K}: y(0)=z, v(0)=\varphi(M)$. For any $t \in \mathbb{R}$ we set

$$
\psi(t)= \begin{cases}y(t), & t \leq 0, \\ \varphi(t), & t \in[0, M] \\ v(t-M), & t \geq M\end{cases}
$$

In view of Lemma $4.1 \psi(\cdot) \in \mathcal{K}$. Therefore, from (7.3) we obtain

$$
\forall k \geq 1 \quad\left\|\Pi_{M} v_{n_{k}}\left(t_{n_{k}}+\cdot\right)-\Pi_{M} \psi(\cdot)\right\|_{C([0, M] ; E)}=\left\|\varphi_{k}-\varphi\right\|_{C([0, M] ; E)} \geq \varepsilon,
$$

that contradicts with (7.4). We reason in the same way when $M=0$.

Thus, the set $D$ constructed in (7.2) is the trajectory attractor in the trajectory space $\mathcal{K}_{+}$ with respect to the topology of $C^{\text {loc }}\left(\mathbb{R}_{+} ; E\right)$.

\section{Auxiliary Properties of the Global and Trajectory Attractors}

Let $A$ be the global attractor from Theorem 5.1, and $D$ the trajectory attractor from Theorem 7.1. From previous sections results we have

$$
\begin{gathered}
\mathcal{A} \text { is a compact set in the space } E ; \\
D \text { is a compact set in the space } C^{\text {loc }}\left(\mathbb{R}_{+} ; E\right) ; \\
D=\Pi_{+} \mathcal{K}=\left\{y \in \mathcal{K}_{+} \mid y(t) \in \mathcal{A} \forall t \in \mathbb{R}_{+}\right\}=\left\{y \in \mathcal{K}_{+} \mid y(0) \in \mathcal{A}\right\},
\end{gathered}
$$

where $\mathcal{K}$ is the family of all complete trajectories of the inclusion $(2.1), \Pi_{+}$is the restriction operator on $\mathbb{R}_{+}$. Moreover,

$$
\begin{gathered}
\mathcal{K} \text { is a compact in the space } C^{\text {loc }}(\mathbb{R} ; E) ; \\
\forall \xi(\cdot) \in \mathcal{K}, \quad \forall t \in \mathbb{R} \xi(t) \in \mathcal{A} ; \\
\forall y_{0} \in \mathcal{A}, \forall t_{0} \in \mathbb{R} \exists y(\cdot) \in \mathcal{K}: \quad y\left(t_{0}\right)=y_{0} .
\end{gathered}
$$

\section{1. "Translation Compactness" of the Trajectory Attractor}

For any $y \in \mathcal{K}$ let us set

$$
\mathscr{H}(y)=\operatorname{cl}_{C^{\operatorname{loc}}(\mathbb{R} ; E)}\{y(\cdot+s) \mid s \in \mathbb{R}\} \subset C^{\text {loc }}(\mathbb{R} ; E) \cap L^{\infty}(\mathbb{R} ; E)
$$

Such family is said to be the hull of function $y(\cdot)$ in $\Xi=C^{\text {loc }}(\mathbb{R} ; E)$.

Definition 8.1. The function $y(\cdot) \in \Xi$ is said to be translation-compact (tr.-c.) in $\Xi$ if the hull $\mathscr{H}(y)$ is compact in $\Xi$. 
Similar constructions for the set of functional parameters that are responsible for nonautonomy of evolution equation are considered, for example, in [46, page 917].

Definition 8.2. The family $\mathcal{U} \subset \Xi$ is said to be translation-compact if $\mathscr{\ell}(\mathcal{U})=\operatorname{cl}_{\Xi}\{y(\cdot+s) \mid y(\cdot)$ $\in \mathcal{U}, s \in \mathbb{R}\}$ is a compact in $\Xi$.

From the autonomy of problems (2.1) and (8.2), the following follows.

Corollary 8.3. $\propto$ is translation-compact set in $\Xi$.

From autonomy of system (2.1), applying the Arzelá-Ascoli compactness criterion (see the proof of Proposition 6.1 from [46]), we obtain the translation compactness criterion for the family $\mathcal{K}$ :

(a) the set $\{y(t) \mid y \in \mathcal{K}\}$ is a compact in $E$ for all $t \in \mathbb{R}$;

(b) there exists a positive function $\alpha(s) \rightarrow 0+(s \rightarrow 0+)$ such that

$$
\left\|y\left(t_{1}\right)-y\left(t_{2}\right)\right\|_{E} \leq \alpha\left(\left|t_{1}-t_{2}\right|\right) \quad \forall y \in \mathcal{K}, \forall t_{1}, t_{2} \in \mathbb{R}
$$

Similarly, if we set $\Xi_{+}=C^{\text {loc }}\left(\mathbb{R}_{+} ; E\right)$, we obtain the following.

Corollary 8.4. $p$ is translation-compact set in $\Xi_{+}$.

\subsection{Stability}

Definition 8.5 (see [43, page 487]). The subset $F \subset E$ is Lyapunov stable if for a given $\varepsilon>0$ there exists $\delta>0$ such that if $D \subset E$ with $\operatorname{dist}(D, F)<\delta$ then $\operatorname{dist}(\mathcal{G}(t, D), F)<\varepsilon$ for all $t \geq 0$.

From [43, page 487], it follows that $F$ is Lyapunov stable if and only if given $\left\{\varphi_{j}(\cdot)\right\}_{j \geq 1} \subset$ $\mathcal{K}_{+}$with $\operatorname{dist}\left(\varphi_{j}(0), F\right) \rightarrow 0, j \rightarrow+\infty$ and $t_{j} \geq 0$ we have $\operatorname{dist}\left(\varphi_{j}\left(t_{j}\right), F\right) \rightarrow 0, j \rightarrow+\infty$.

Definition 8.6 (see [43, page 487]). The subset $F$ is uniformly asymptotically stable if $F$ is Lyapunov stable and it is locally attracting, that is, if it attracts a neighborhood of $F$ (see [43, page 482]).

Note that bounded attracting set is locally attracting one.

Corollary 8.7. The global attractor \& from Theorem 5.1 is uniformly asymptotically stable.

Proof. The proof follows from the definition of $\mathcal{G},[43$, Theorem 6.1], properties of solutions from Lemma 3.2, Theorem 4.6, and from the autonomy of problem (2.1).

Similar results are true for sets $D$ and $\mathcal{K}$ in corresponding extended phase spaces.

\subsection{Connectedness}

Definition 8.8 (see [43, page 485]). The $m$-semiflow $\mathcal{G}$ has Kneser's property, if $\mathcal{G}(t, z)$ is connected for each $z \in E, t \geq 0$. 
Corollary 8.9. If $\mathcal{G}$ has Kneser's property, then $\mathbb{A}$ is connected.

Proof. The proof follows from [43, Corollary 4.3], Lemma 3.2, and from the connectedness of the phase space $E$.

Note that Kneser's property for $\mathcal{G}$ can be checked by different way (see, e.g., [35, 36, 47]). In order to do that as a rule, it is required an auxiliary regularity of interaction functions. In the general case, Kneser's property for problem (2.1) can be checked using the method of proof from [47, Theorem 5], where we can consider Yosida approximation instead the proposed approximation.

Corollary 8.10. If $\mathcal{K}_{+}$is connected, then $\mathcal{K} \subset C^{\text {loc }}(\mathbb{R} ; E)$ is connected, and $D \subset C^{\text {loc }}\left(\mathbb{R}_{+} ; E\right)$ is connected.

Proof. The proof is trivial.

\section{Applications}

As an application we consider the hemivariational inequality of hyperbolic type with multidimensional "reaction-displacement" law [19] (see Problem (1.1)-(1.6), undermentioned above conditions). From results of previous sections, it follows the next result.

Corollary 9.1. The $m$-semiflow $\mathcal{G}$ constructed on all generalized solutions of (1.1)-(1.6) has the compact invariant global attractor $\mathbf{A}$. For all generalized solutions of $(1.1)-(1.6)$, defined on $[0,+\infty)$, there exists the trajectory attractor $D$, and we have

$$
\mathcal{A}=D(0)=\{y(0) \mid y \in \mathcal{K}\}, \quad D=\Pi_{+} \mathcal{K},
$$

where $\mathcal{K}$ is the family of all complete trajectories of corresponding differential-operator inclusion (2.1) in $C^{\text {loc }}(\mathbb{R} ; E) \cap L_{\infty}(\mathbb{R} ; E)$. Moreover, global attractors are equal in the sense of $[44$, Definition 6 , page 88] as well as in the sense of [42, Definition 2.2, page 182].

For each $\psi \in \mathcal{K}$, the limit sets:

$$
\begin{aligned}
& \alpha(\psi)=\left\{z \in E \mid \psi\left(t_{j}\right) \longrightarrow z \text { for some sequence } t_{j} \longrightarrow-\infty\right\}, \\
& \omega(\psi)=\left\{z \in E \mid \psi\left(t_{j}\right) \longrightarrow z \text { for some sequence } t_{j} \longrightarrow+\infty\right\},
\end{aligned}
$$

are connected subsets of $Z(\mathcal{G})$ on which $\mho$ is constant.

Thus, all statements of previous sections are true for all generalized solutions of problem (1.1)-(1.6). In particular, all displacements and velocities are "attracted" as $t \rightarrow$ $+\infty$ to all complete (defined on the entire time axis), globally bounded trajectories of corresponding "generalized" problem (2.1), which belong to compact sets in corresponding phase and extended phase spaces. Note that approaches proposed in works [42,44] are based on properties of solutions of evolution objects. Our approaches are based on properties of interaction functions from (2.1) and properties of phase spaces. 


\section{Acknowledgments}

The authors would like to express their gratitude to Professor M. Z. Zgurovsky, Rector of NTUU "KPI", Director of IASA NAS of Ukraine for providing excellent research facilities.

\section{References}

[1] S. Migórski, "Dynamic hemivariational inequalities in contact mechanics," Nonlinear Analysis: Theory, Methods and Applications, vol. 63, no. 5-7, pp. e77-e86, 2005.

[2] Z. Naniewicz and P. D. Panagiotopoulos, Mathematical Theory of Hemivariational Inequalities and Applications, vol. 188 of Monographs and Textbooks in Pure and Applied Mathematics, Marcel Dekker, New York, NY, USA, 1995.

[3] P. D. Panagiotopoulos, Inequality Problems in Mechanics and Applications. Convex and Nonconvex Energy Functions, Birkhäuser, Boston, Mass, USA, 1985.

[4] I. D. Chueshov, "Global attractors for non-linear problems of mathematical physics," Russian Mathematical Surveys, vol. 48, no. 3, pp. 135-162, 1993.

[5] F. H. Clarke, Optimization and Nonsmooth Analysis, Canadian Mathematical Society Series of Monographs and Advanced Texts, John Wiley \& Sons, New York, NY, USA, 1983.

[6] Yu. A. Dubinskii, "High order nonlinear parabolic equations," Journal of Soviet Mathematics, vol. 56, no. 4, pp. 2557-2607, 1991.

[7] G. Duvaut and J.-L. Lions, Les inéquations en mécanique et en physique, Travaux et Recherches Mathématiques, No. 21, Dunod, Paris, France, 1972.

[8] H. Gajewski, K. Gröger, and K. Zacharias, Nichtlineare Operatorgleichungen und Operatordifferentialgleichungen, Mathematische Lehrbücher und Monographien, II. Abteilung, Mathematische Monographien, Band 38, Akademie, Berlin, Germany, 1974.

[9] D. Goeleven, M. Miettinen, and P. D. Panagiotopoulos, "Dynamic hemivariational inequalities and their applications," Journal of Optimization Theory and Applications, vol. 103, no. 3, pp. 567-601, 1999.

[10] S. Hu and N. S. Papageorgiou, Handbook of Multivalued Analysis. Vol. I: Theory, vol. 419 of Mathematics and Its Applications, Kluwer Academic Publishers, Dordrecht, The Netherlands, 1997.

[11] S. Hu and N. S. Papageorgiou, Handbook of Multivalued Analysis. Vol. II: Applications, vol. 500 of Mathematics and Its Applications, Kluwer Academic Publishers, Dordrecht, The Netherlands, 2000.

[12] P. Kalita, "Decay of energy for second-order boundary hemivariational inequalities with coercive damping," Nonlinear Analysis: Theory, Methods E Applications, vol. 74, no. 4, pp. 1164-1181, 2011.

[13] P. O. Kasyanov, V. S. Melnik, and S. Toscano, "Solutions of Cauchy and periodic problems for evolution inclusions with multi-valued $w_{10}$-pseudomonotone maps," Journal of Differential Equations, vol. 249, no. 6, pp. 1258-1287, 2010.

[14] P. O. Kasyanov, "Multivalued dynamics of solutions of an autonomous differential-operator inclusion with pseudomonotone nonlinearity," Cybernetics and Systems Analysis, vol. 47, no. 5, pp. 800-811, 2011.

[15] K. Kuttler, "Non-degenerate implicit evolution inclusions," Electronic Journal of Differential Equations, no. 34, pp. 1-20, 2000.

[16] O. A. Ladyzhenskaya, Mathematical Problems of the Dynamics of Viscous Incompressible Fluids, Fizmatgiz, Moscow, Russia, 1961.

[17] J.-L. Lions, Quelques méthodes de résolution des problèmes aux limites non linéaires, Dunod, Paris, France, 1969.

[18] Z. Liu and S. Migórski, "Noncoercive damping in dynamic hemivariational inequality with application to problem of piezoelectricity," Discrete and Continuous Dynamical Systems B, vol. 9, no. 1, pp. 129-143, 2008.

[19] S. Migórski, "Boundary hemivariational inequalities of hyperbolic type and applications," Journal of Global Optimization, vol. 31, no. 3, pp. 505-533, 2005.

[20] S. Migorski, "Evolution hemivariational inequalities with applications," in Handbook of Nonconvex Analysis and Applications, D. Y. Gao and D. Motreanu, Eds., chapter 8, pp. 409-473, International Press, Somerville, Mass, USA, 2010.

[21] S. Migorski, "Existence of solutions to nonlinear second order evolution inclusions without and with impulses," Dynamics of Continuous, Discrete E Impulsive Systems B, vol. 18, no. 4, pp. 493-520, 2011.

[22] R. Temam and A. Miranville, Mathematical Modeling in Continuum Mechanics, Cambridge University Press, Cambridge, UK, 2001.

[23] S. T. Mohyud-Din, M. A. Noor, and K. I. Noor, "Some relatively new techniques for nonlinear problems," Mathematical Problems in Engineering, vol. 2009, Article ID 234849, 25 pages, 2009. 
[24] M. Aslam Noor, "Some developments in general variational inequalities," Applied Mathematics and Computation, vol. 152, no. 1, pp. 199-277, 2004.

[25] M. A. Noor, “On iterative methods for solving a system of mixed variational inequalities," Applicable Analysis, vol. 87, no. 1, pp. 99-108, 2008.

[26] M. A. Noor, "On a system of general mixed variational inequalities," Optimization Letters, vol. 3, no. 3, pp. 437-451, 2009.

[27] M. A. Noor, "On an implicit method for nonconvex variational inequalities," Journal of Optimization Theory and Applications, vol. 147, no. 2, pp. 411-417, 2010.

[28] M. A. Noor, "Extended general variational inequalities," Applied Mathematics Letters, vol. 22, no. 2, pp. 182-186, 2009.

[29] M. A. Noor, K. I. Noor, and Z. Huang, "Bifunction hemivariational inequalities," Journal of Applied Mathematics and Computing, vol. 35, no. 1-2, pp. 595-605, 2011.

[30] M. A. Noor, K. I. Noor, and E. Al-Said, "Iterative methods for solving nonconvex equilibrium variational inequalities," Applied Mathematics E Information Sciences, vol. 6, no. 1, pp. 65-69, 2012.

[31] M. A. Noor, "Some aspects of extended general variational inequalities," Abstract and Applied Analysis. In press.

[32] P. D. Panagiotopoulos, "Hemivariational inequalities and fan-variational inequalities. New applications and results," Atti del Seminario Matematico e Fisico dell'Università di Modena, vol. 43, no. 1, pp. 159-191, 1995.

[33] G. R. Sell and Y. You, Dynamics of Evolutionary Equations, vol. 143 of Applied Mathematical Sciences, Springer, New York, NY, USA, 2002.

[34] R. Temam, Infinite-Dimensional Dynamical Systems in Mechanics and Physics, vol. 68 of Applied Mathematical Sciences, Springer, New York, NY, USA, 1988.

[35] A. A. Tolstonogov, "Solutions of Evolution Inclusions. I," Sibirskiŭ Matematicheskiu Zhurnal, vol. 33, no. 3, pp. 161-174, 1992.

[36] A. A. Tolstonogov and Ya. I. Umanskiŭ, "Solutions of evolution inclusions. II," Sibirskiŭ Matematicheskiŭ Zhurnal, vol. 33, no. 4, pp. 163-174, 1992.

[37] N. V. Zadoyanchuk and P. O. Kasyanov, "The Faedo-Galerkin method for second-order evolution inclusions with $W_{\lambda}$-pseudomonotone mappings," Ukrainian Mathematical Journal, vol. 61, no. 2, pp. 195-213, 2009.

[38] N. V. Zadoyanchuk and P. O. Kasyanov, "Analysis and control of second-order differential-operator inclusions with +-coercive damping," Cybernetics and Systems Analysis, vol. 46, no. 2, pp. 305-313, 2010.

[39] M. Z. Zgurovsky, V. S. Mel'nik, and P. O. Kasyanov, Evolution Inclusions and Variation Inequalities for Earth Data Processing I. Operator Inclusions and Variation Inequalities for Earth Data Processing, vol. 24 of Advances in Mechanics and Mathematics, Springer, Berlin, Germany, 2011.

[40] M. Z. Zgurovsky, V. S. Mel'nik, and P. O. Kasyanov, Evolution Inclusions and Variation Inequalities for Earth Data Processing II. Differential-Operator Inclusions and Evolution Variation Inequalities for Earth Data Processing, vol. 25 of Advances in Mechanics and Mathematics, Springer, Berlin, Germany, 2011.

[41] J. M. Ball, "Global attractors for damped semilinear wave equations," Discrete and Continuous Dynamical Systems A, vol. 10, no. 1-2, pp. 31-52, 2004.

[42] M. I. Vishik and V. V. Chepyzhov, "Trajectory and global attractors of the three-dimensional NavierStokes system," Matematicheskie Zametki, vol. 71, no. 2, pp. 194-213, 2002.

[43] J. M. Ball, "Continuity properties and global attractors of generalized semiflows and the NavierStokes equations," Journal of Nonlinear Science, vol. 7, no. 5, pp. 475-502, 1997.

[44] V. S. Melnik and J. Valero, "On attractors of multivalued semi-flows and differential inclusions," SetValued Analysis, vol. 6, no. 1, pp. 83-111, 1998.

[45] O. V. Kapustyan and J. Valero, "Comparison between trajectory and global attractors for evolution systems without uniqueness of solutions," International Journal of Bifurcation and Chaos in Applied Sciences and Engineering, vol. 20, no. 9, pp. 2723-2734, 2010.

[46] V. V. Chepyzhov and M. I. Vishik, "Evolution equations and their trajectory attractors," Journal de Mathématiques Pures et Appliquées. Neuvième Série, vol. 76, no. 10, pp. 913-964, 1997.

[47] J. Valero and A. Kapustyan, "On the connectedness and asymptotic behaviour of solutions of reactiondiffusion systems," Journal of Mathematical Analysis and Applications, vol. 323, no. 1, pp. 614-633, 2006. 


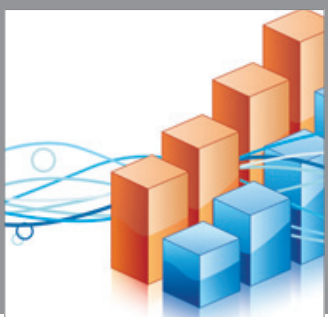

Advances in

Operations Research

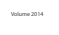

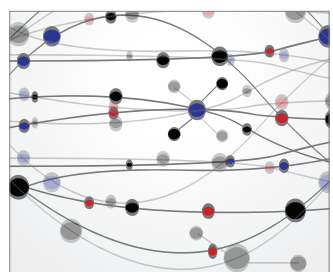

\section{The Scientific} World Journal
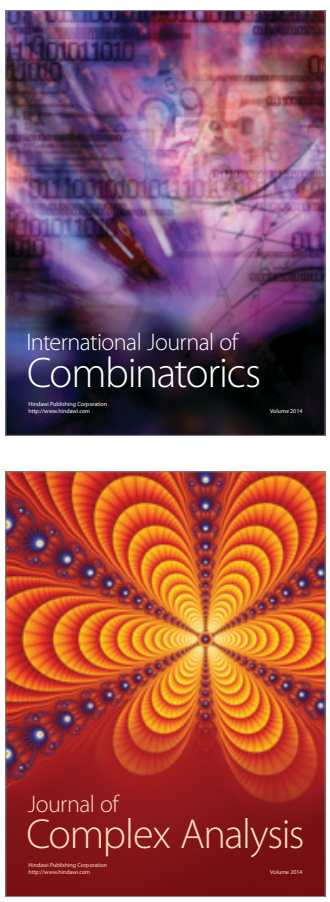

International Journal of

Mathematics and

Mathematical

Sciences
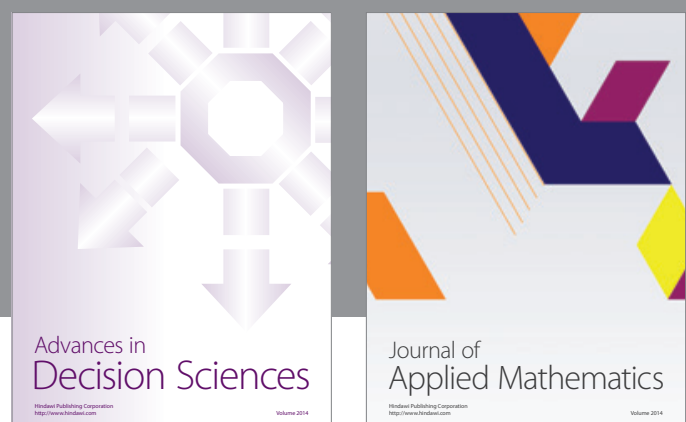

Journal of

Applied Mathematics
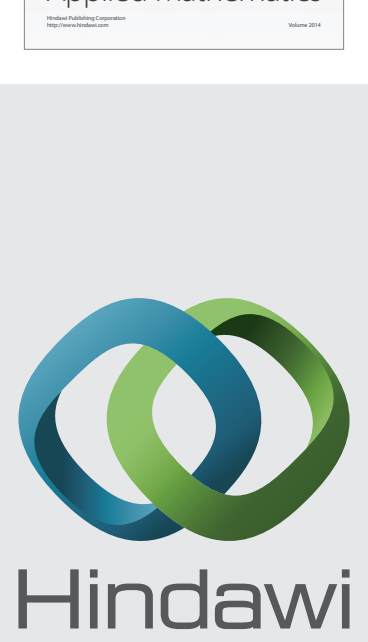

Submit your manuscripts at http://www.hindawi.com
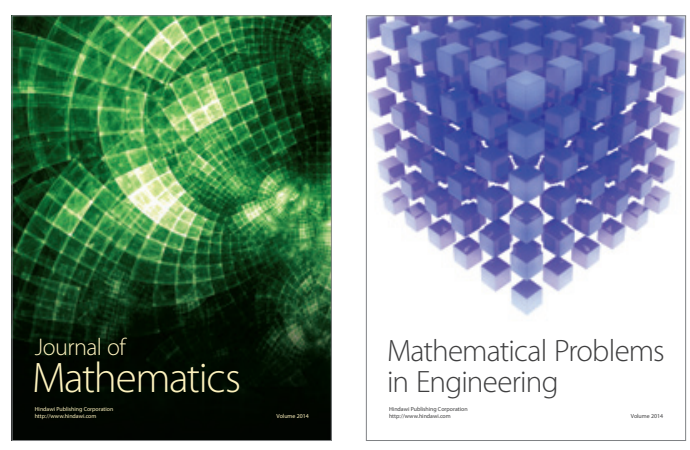

Mathematical Problems in Engineering
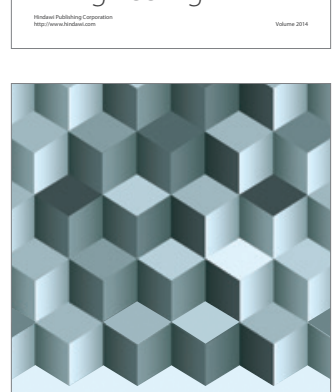

Journal of

Function Spaces
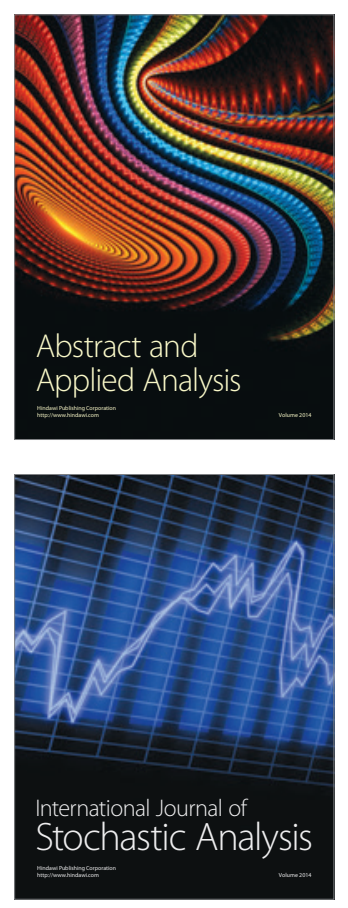

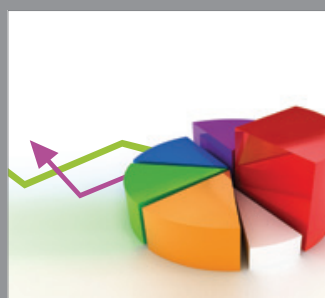

ournal of

Probability and Statistics

Promensencen
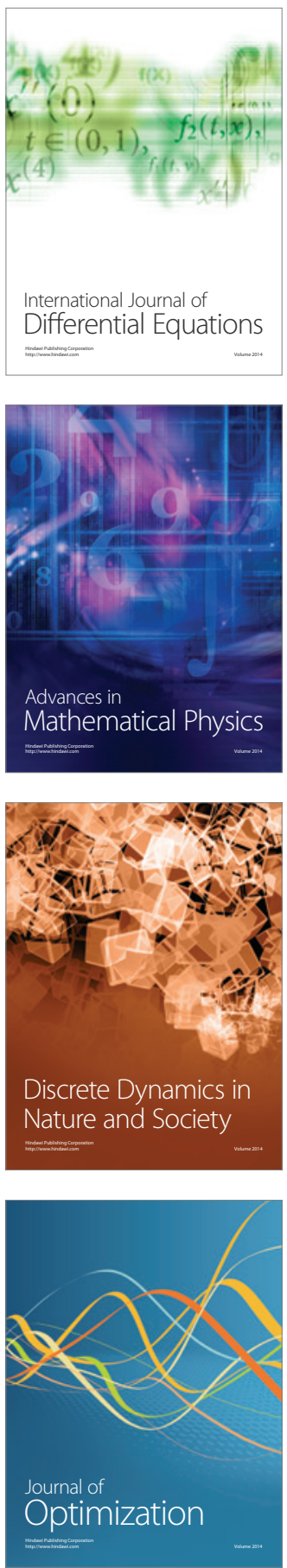